\title{
Complications of Transcutaneous Protheses - A Systematic Review of Publications Over the Past 10 Years
}

\author{
Marcos Antonio de Souza ${ }^{1}$ Santiago Luis Vallejos Riart ${ }^{2}$ Stephanie Rugeri de Souza ${ }^{1}$ \\ Rubens de Brito $^{10}$ Ricardo Ferreira Bento ${ }^{10}$
}

${ }^{1}$ Department of Otorhinolaryngology, Universidade de São Paulo (USP), São Paulo, SP, Brazil

2 Department of Otorhinolaryngology, Universidad Nacional de Asunción (UNA), San Lorenzo, Central, Paraguay

\begin{abstract}
Address for correspondence Marcos Antonio de Souza, MD, Departamento de Otorrinolaringologia, USP, Rua, Av. Dr. Enéas Carvalho de Aguiar, 255 - Cerqueira César, São Paulo, SP 05403-000,
\end{abstract} Brazil (e-mail: marcosantonio.med@gmail.com).

Int Arch Otorhinolaryngol 2022;26(3):e505-e512.

\begin{abstract}
\section{Keywords}

- Baha Attract

- BoneBridge

- Sophono

- bone-conduction implant

- transcutaneous

Introduction Bone conduction implants, responsible for transmitting sound from an external processor to the inner ear, can be divided into active and passive, depending on the vibratory stimulus location. The use of transcutaneous device has increased, given its aesthetic appeal, the complications and limitations of percutaneous devices, and patient's treatment adherence, focusing mainly on efficacy. However, various complications are associated with the use of transcutaneous prosthesis, which can often be serious.

Objective To approach the literature on complications involving transcutaneous bone-anchored prostheses through a systematic review of articles published in the past 10 years (2011-2021).

Data Synthesis The MEDLINE, EMBASE, Scopus, and Cochrane Library databases were searched. All articles written in English reporting on currently available transcutaneous prosthesis implantation and its complications were selected. Studies on both children and adults were included. The data on complications were extracted, and complications were classified as minor or major and associated to each device used. Thirty-seven articles were included in the study, of which 14 were prospective cohort studies, 22 were retrospective case series, and 1 was a case report. Most studies (18) included both adults and children. Moreover, 901 implantations were performed, of which 552 implanted Baha Attract (Cochlear Ltd., Sydney, Australia), 244 implanted BoneBridge (MED-EL, Innsbruck, Austria), and 105 implanted Sophono (Sophono Inc., Boulder, CO, USA]). Furthermore, 192 adverse events were reported (total complication rate, $21.3 \%$ ), with 161 minor complications (84.3\%) and 31 major complications (16.1\%). Conclusion Transcutaneous prosthesis is an audiological alternative with fewer complications than percutaneous prosthesis. However, its indication should be judicious because complications are common, and although most complications are minor, serious infections requiring explantation may develop.
\end{abstract}

received

July 6,2021

accepted after revision

October 20, 2021

published online

February 4, 2022
DOI https://doi.org/ 10.1055/s-0042-1742352. ISSN 1809-9777.

\footnotetext{
(C) 2022. Fundação Otorrinolaringologia. All rights reserved.

This is an open access article published by Thieme under the terms of the Creative Commons Attribution-NonDerivative-NonCommercial-License, permitting copying and reproduction so long as the original work is given appropriate credit. Contents may not be used for commercial purposes, or adapted, remixed, transformed or built upon. (https://creativecommons.org/ licenses/by-nc-nd/4.0/) Thieme Revinter Publicações Ltda., Rua do Matoso 170, Rio de Janeiro, RJ, CEP 20270-135, Brazil
} 


\section{Introduction}

The conduction of sound through bone was already known in the 2 nd century AD by Claudius Galenus (131-200) and was first described and applied by Girolamo Cardano (15101580). ${ }^{1}$ Following this principle, Ludwig van Beethoven (1770-1827), with an advanced hearing loss in his last years, used a stick with one end resting on his piano and the other between his teeth to transmit sound to his ears. ${ }^{2}$

Bone-conduction implants transmit auditory stimuli from an external audio processor to the inner ear. Hearing is produced by conducting sound waves through the skull bones to the cochlea, bypassing the outer and middle ears. These devices have been shown to effectively treat conductive and mixed hearing loss and unilateral deafness. They are a better treatment option than reconstructive surgery for patients with bilateral aural atresia, presenting great advantages in terms of audiological outcomes and

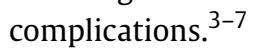

Currently, bone-conduction devices available on the market can be classified into two main categories: percutaneous devices, which penetrate the skin and have a direct connection to the implant (Baha Connect [Cochlear Bone-Anchored Solutions AB, Mölnlycke, Sweden] and Ponto [Oticon Medical $A B$, Askim, Sweden]), and transcutaneous devices, which communicate with the implant through intact skin and are held together by magnets. Transcutaneous devices, in turn, are divided into passive bone-conduction systems, in which vibration is transmitted from the processor to the implant through the skin through a magnet system (Sophono Alpha [Sophono Inc., Boulder, CO, USA] and Baha Attract [Cochlear Bone-Anchored Solutions AB, Mölnlycke, Sweden]), and active systems, in which the implant directly generates vibration and applies it to the bone (BoneBridge [MED-EL, Innsbruck, Austria] and Osia [Cochlear Ltd., Sydney, Australia]). ${ }^{8,9}$

Percutaneous implants have been well studied and have excellent audiometric results. However, many patients prefer not to undergo this surgery for aesthetic reasons related to the percutaneous abutment or due to possible complications, such as infection, skin overgrowth at the external site of the implant, and skin rupture or necrosis, which can lead to loss of implant. ${ }^{10-14}$

In the last decade, the use of transcutaneous devices has increased, given the complications and limitations of percutaneous devices, in addition to the aesthetic appeal associated with transcutaneous prostheses and patient adherence to treatment, focusing mainly on efficacy. ${ }^{15,16}$ These benefits are also supported by studies that suggest that fewer complications occur with transcutaneous models than with percutaneous ones. However, hearing results still have worse performance due to sound cushioning through the skin. ${ }^{17,18}$

The present study aims to systematically review the articles published in the past 10 years and listed in the available databases presenting patients with transcutaneous bone-conduction devices and the complications associated with these devices to document these lesions and assist in the selection of these devices.

\section{Review of Literature}

The present study includes a systematic review of the postoperative complications related to transcutaneous bone-anchored prostheses, through an analysis of articles addressing this topic that were published in the past 10 years (2011-2021).

\section{Search Methodology}

Our institution's health sciences research librarian was consulted, and a structured query was performed in May 2021. The analyzed databases were PUBMED, EMBASE, Cochrane Library, LILACS, SCOPUS, and Web of Science.

The query terms, with their respective combinations, were hearing aid OR bone conduction OR transcutaneous AND complications (Baha Attract OR Sophono OR BoneBridge) AND bone conduction implant $\mathrm{OR}$ *bone-conduction implant, with filters applied for publications from the previous 10 years and for study design.

The query results for each database were compiled, and, initially, duplicate articles were identified and removed. All abstracts were reviewed by two authors (M. A. S. and S. L. V. R.) regarding their relevance and alignment with the subject under study.

The full text of the relevant studies was reviewed, and the reference lists were cross-checked to identify important additional studies. Disagreement regarding the inclusion of individual studies was resolved after a debate between the two reviewers.

\section{Inclusion Criteria}

The inclusion criteria consisted of articles published only in English, addressing the implantation of active and passive transcutaneous prostheses (BoneBridge, Sophono, or Baha Attract) and the complications derived from the implantation. The reason for choosing these specific implants relates to the vast literature available about them, given the time they already have been on the market. Implants that were launched in the previous 5 years, on average, and those with only sporadic use or lacking more scientific evidence were excluded. Studies with both adult and pediatric populations were selected.

All selected studies had their abstracts read in full, and those that addressed the performance of a transcutaneous prosthesis, the existence of complications related to the prosthesis, and studied a human population were selected for full-text reading. Studies that exclusively addressed percutaneous prostheses, cochlear implants, stapedotomy prostheses, and those that did not delimit possible complications were excluded. There was no limitation on the level of evidence.

After reading the articles selected in full, the two authors rated the complications. Complications were considered to be major when they required active medical or surgical treatment, such as new surgical procedures, or when their existence led to the cessation of use of the device. Minor complications consisted of minor soft-tissue reactions, including pain and erythema, that may have reduced the use of 
the device, but did not prevent that use altogether. Such complications were necessarily corrected only with conservative measures.

The exclusion criteria comprised articles that were not available in English, those based on laboratory or cadaver models, 3D reproductions, review articles without additional patients reported, and articles reporting preliminary results for later publications that described the same cohort of patients. Articles that addressed transcutaneous prostheses but had no reports of complications were also excluded.

\section{Study Selection}

The delimited query strategy yielded 274 articles (-Fig. 1). After filtering out duplicate articles, 231 articles remained for review and abstract reading. Based on the analysis of the abstracts, 93 articles were selected for full-text reading and analysis, of which 56 were excluded for the following reasons: 26 did not define in their text the occurrence of complications, either minor or major, or were only descriptive reports published as book chapters; 13 addressed the occurrence of complications of percutaneous or other prostheses that were not included in the present study design; 6 articles were systematic reviews that did not add additional patients; 8 articles were not available in English; and 3 were abstracts without an associated published manuscript. The 37 selected articles are available in - Table 1.

The included studies comprised 14 prospective cohort studies, 22 retrospective case series, and 1 case report. Most studies (18) included both adult and pediatric patients, while

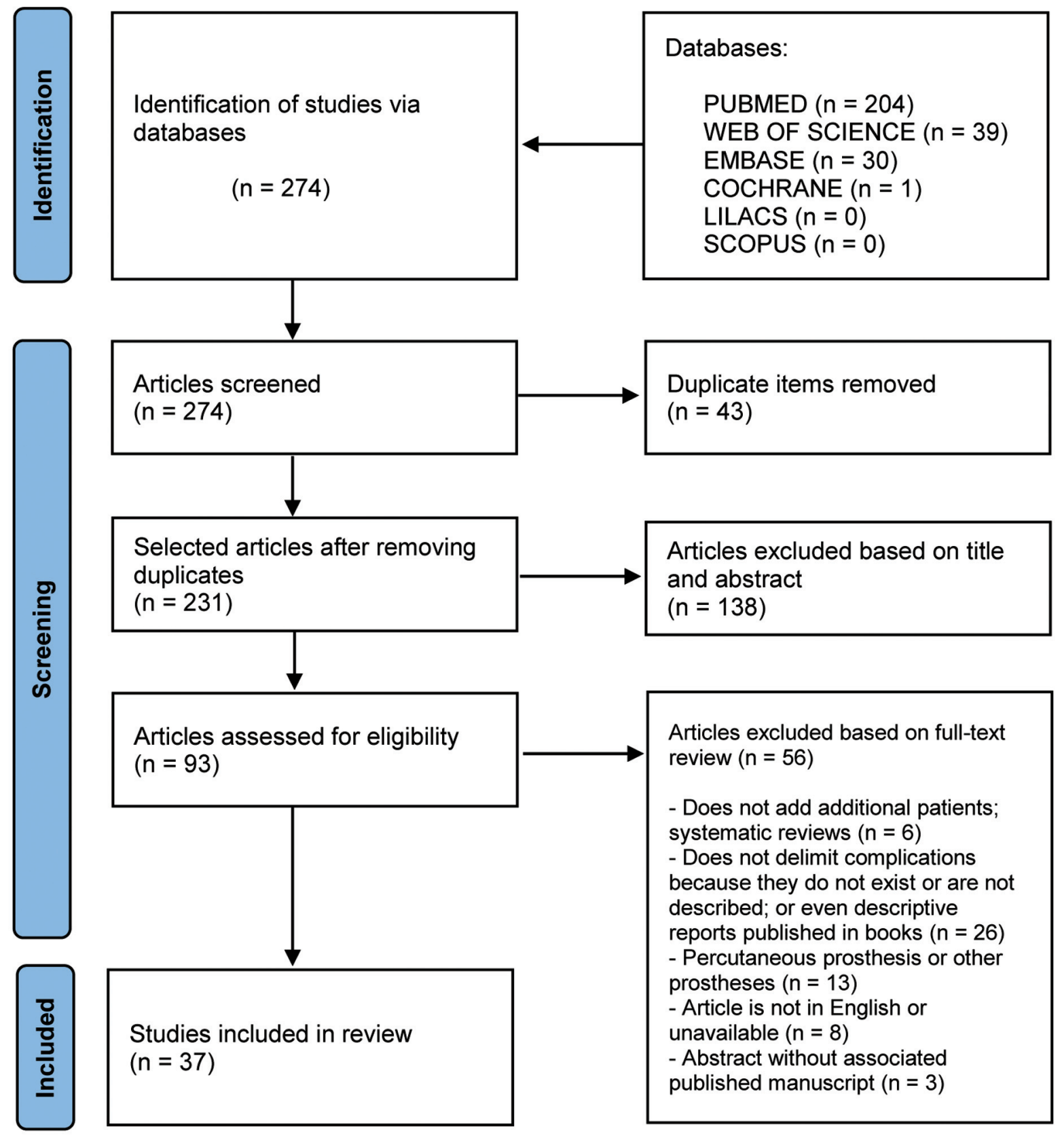

Fig. 1 Systematic review. 


\begin{tabular}{|c|c|c|c|c|c|c|c|c|c|c|c|c|c|c|c|c|c|c|c|}
\hline 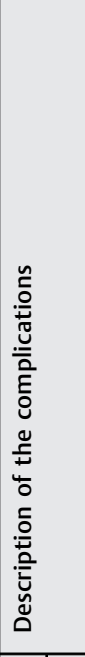 & 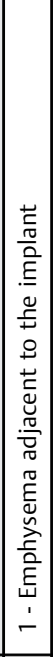 & 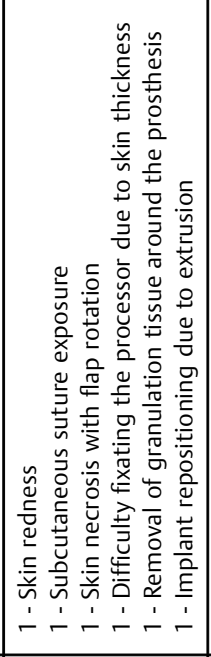 & 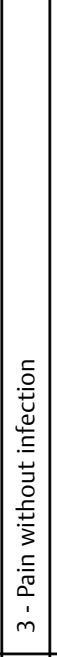 & 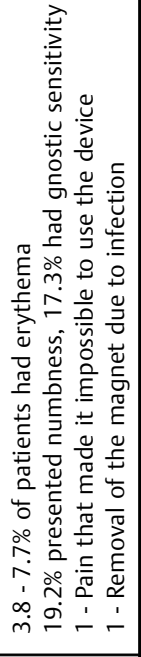 & 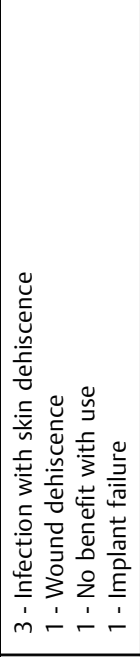 & 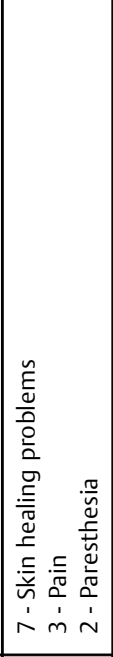 & 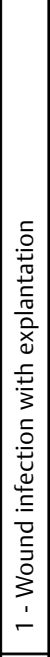 & 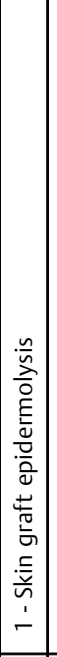 & 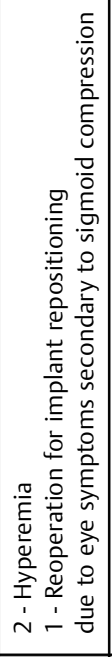 & 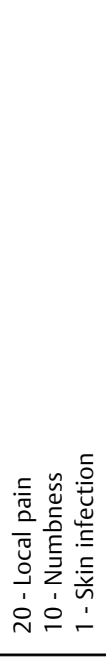 & 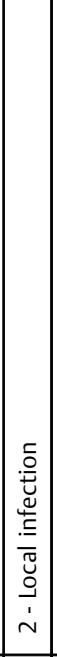 & 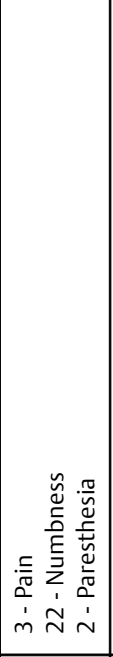 & 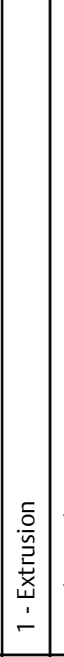 & 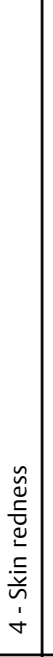 & 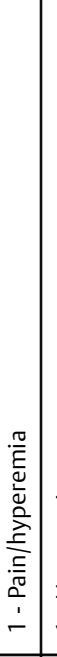 & 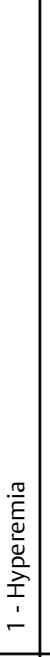 & 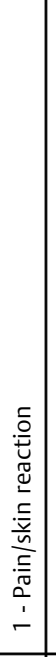 & 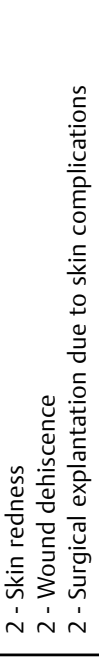 & 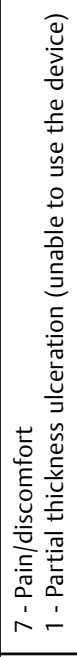 \\
\hline 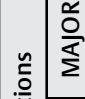 & 0 & $\sigma$ & 0 & $\sim$ & 0 & 0 & - & 0 & - & - & 0 & 0 & - & 0 & $\circ$ & 0 & 0 & $\sim$ & $m$ \\
\hline 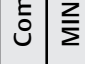 & - & $\sim$ & $m$ & 1 & 0 & $\simeq$ & 0 & -1 & $\sim$ & mे & $\sim$ & 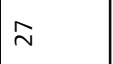 & 0 & $\nabla$ & - & - & - & $\nabla$ & $r$ \\
\hline 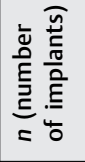 & - & $\bar{m}$ & $\nabla$ & 节 & t & mे & $\approx$ & $\circ$ & $\stackrel{\Xi}{ }$ & in & & $\stackrel{\stackrel{\sim}{\simeq}}{\simeq}$ & $\stackrel{\sim}{\sim}$ & $\stackrel{ \pm}{\sim}$ & $\circ$ & $\circ$ & in & $\stackrel{\Perp}{\sim}$ & m \\
\hline$\stackrel{\mathscr{\nu}}{\bar{\Delta}}$ & 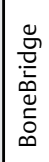 & 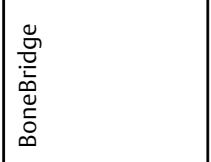 & 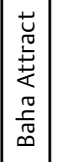 & 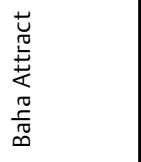 & 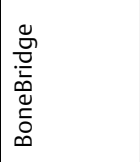 & 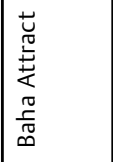 & 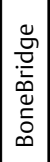 & 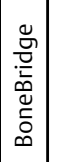 & 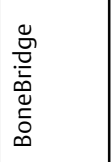 & 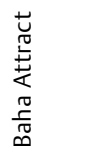 & 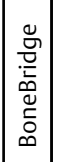 & 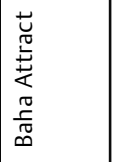 & $\begin{array}{l}\tilde{g} \\
\bar{\sigma} \\
\overline{0} \\
0 \\
\overline{0} \\
0 \\
0\end{array}$ & 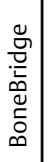 & 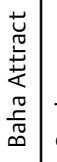 & 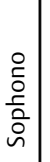 & 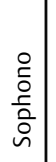 & 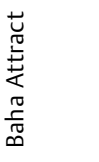 & 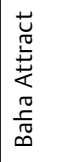 \\
\hline
\end{tabular}

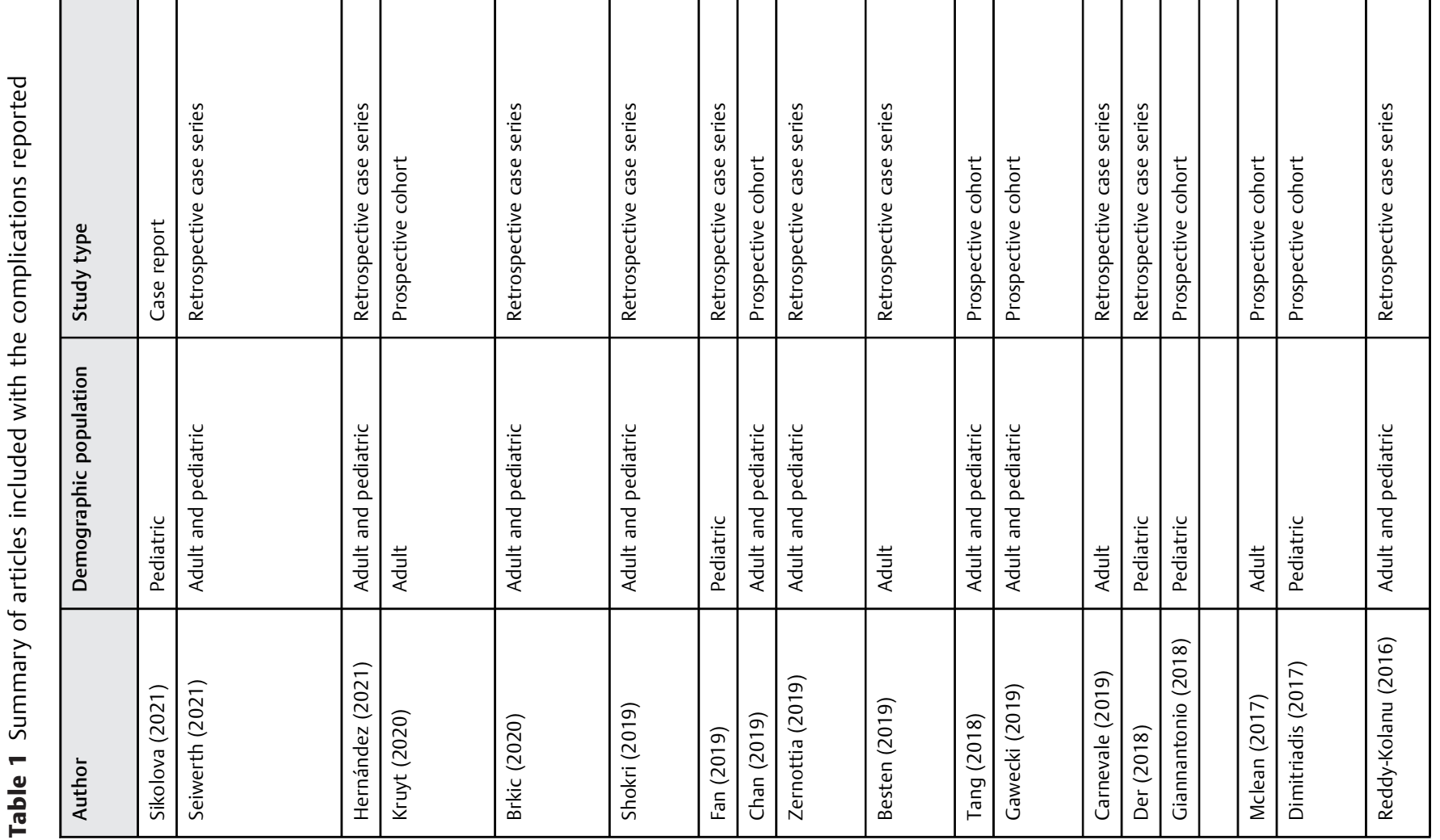




\begin{tabular}{|c|c|c|c|c|c|c|c|c|c|c|c|c|c|c|c|c|c|c|c|c|c|}
\hline 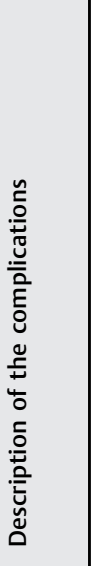 & 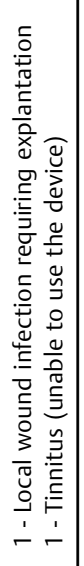 & 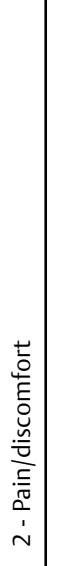 & 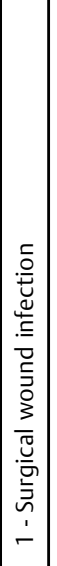 & 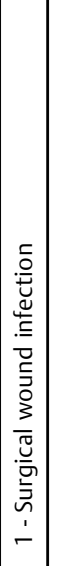 & 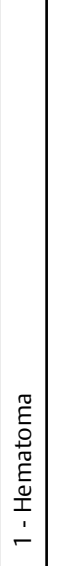 & 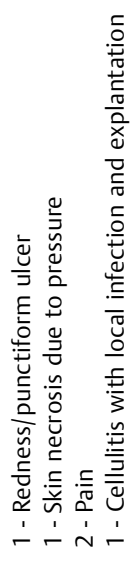 & 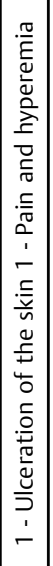 & 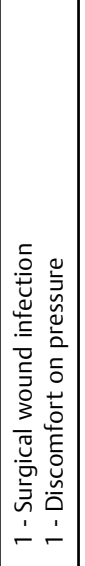 & 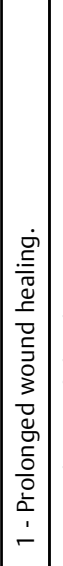 & 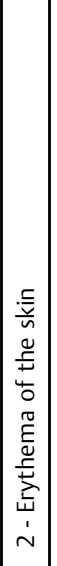 & 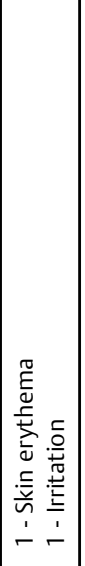 & 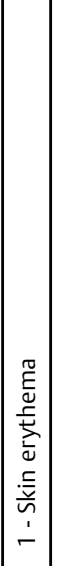 & 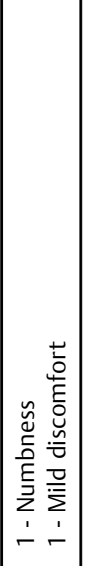 & 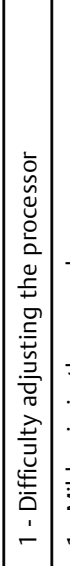 & 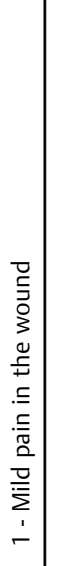 & 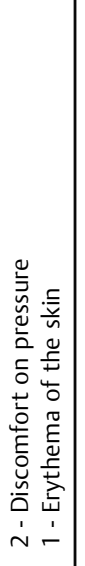 & 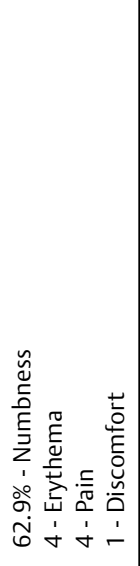 & 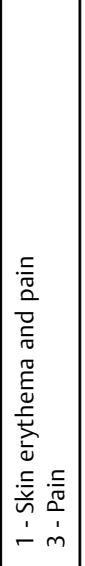 & 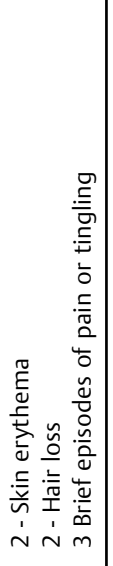 & 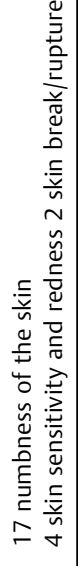 & 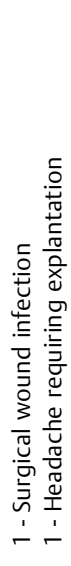 \\
\hline 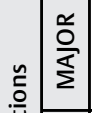 & & 0 & 0 & 0 & 0 & - & $\sim$ & 0 & - & 0 & 0 & 0 & 0 & - & 0 & 0 & 0 & 0 & 0 & $\sim$ & $\sim$ \\
\hline 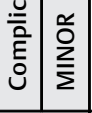 & & $\sim$ & - & - & - & $\nabla$ & 0 & $\sim$ & 0 & $\sim$ & $\sim$ & - & $\sim$ & 0. & - & $m$ & $\sigma$ & $\sigma$ & $\wedge$ & $\bar{\sim}$ & 0 \\
\hline 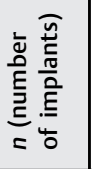 & & a & $\simeq$ & $m$ & $m$ & $\stackrel{\Xi}{ }$ & 0 & in & 0 & 0 & $\stackrel{\sim}{\sim}$ & $\circ$ & ㄱ. & $\stackrel{\sim}{\circ}$ & $\circ$ & $\nabla$ & $\bar{N}$ & $\stackrel{\bullet}{\circ}$ & $\stackrel{\text { n }}{\simeq}$ & $\stackrel{\stackrel{n}{\circ}}{\circ}$ & 0 \\
\hline$\stackrel{\mathscr{u}}{\bar{\Xi}}$ & & 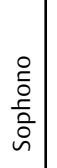 & 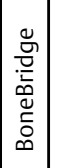 & 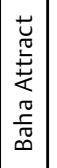 & 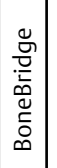 & $\begin{array}{l}\stackrel{\circ}{\circ} \\
\stackrel{\circ}{\circ} \\
\stackrel{\circ}{\circ} \\
\stackrel{\sim}{n}\end{array}$ & 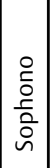 & 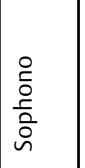 & 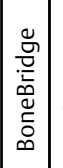 & 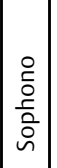 & 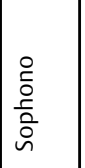 & 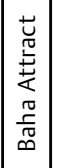 & 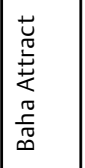 & 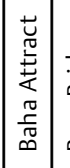 & 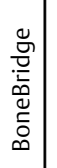 & 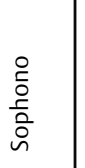 & 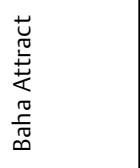 & 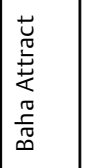 & $\begin{array}{l}\stackrel{\circ}{0} \\
\text { 은 } \\
\dot{\hat{~}}\end{array}$ & 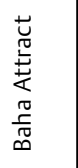 & 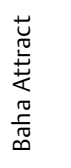 \\
\hline 总 & & 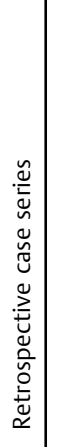 & 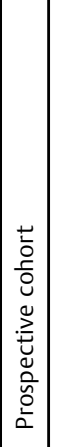 & 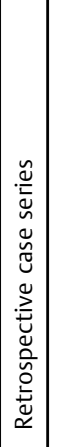 & 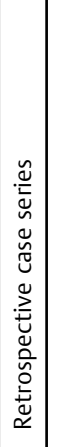 & 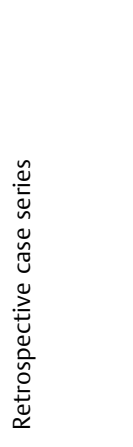 & 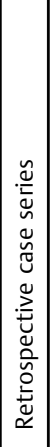 & 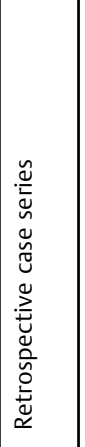 & 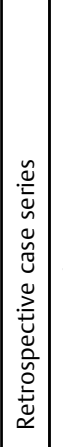 & 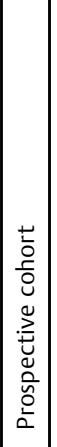 & 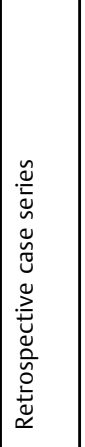 & 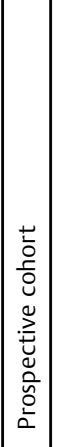 & 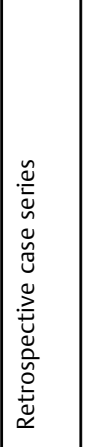 & 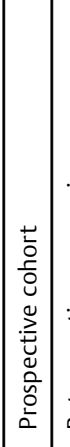 & 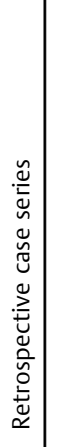 & & 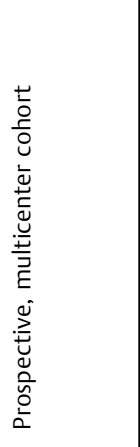 & 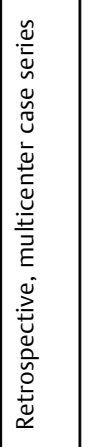 & 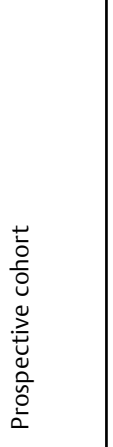 & 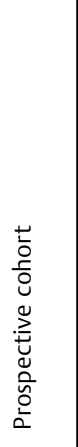 & 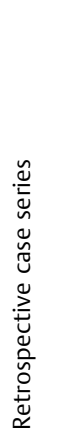 \\
\hline 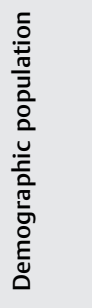 & & 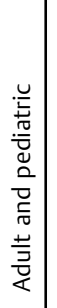 & $\mid$\begin{tabular}{|l} 
\\
$\frac{\Delta}{\frac{3}{4}}$ \\
\end{tabular} & 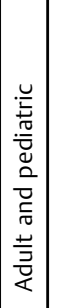 & 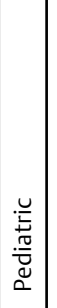 & 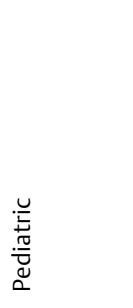 & 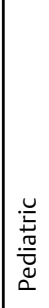 & 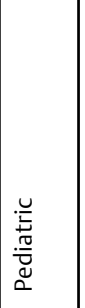 & $\frac{+}{\frac{\Delta}{3}}$ & 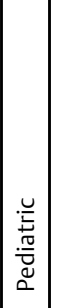 & 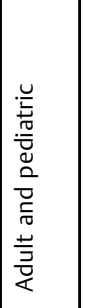 & 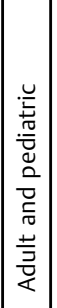 & 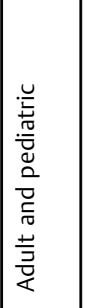 & 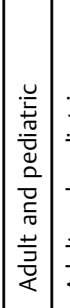 & 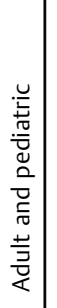 & & $\frac{\frac{\Delta}{5}}{\frac{y}{4}}$ & 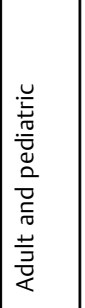 & 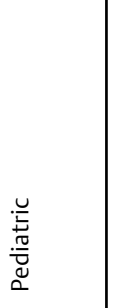 & 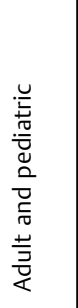 & 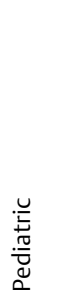 \\
\hline $\begin{array}{l}\text { 高 } \\
\text { 妾 }\end{array}$ & & 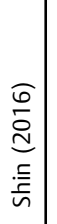 & 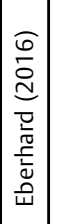 & 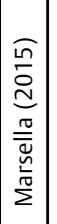 & 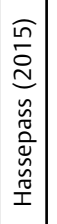 & 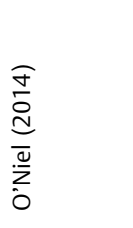 & 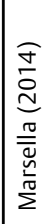 & 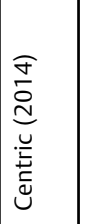 & 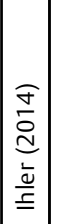 & 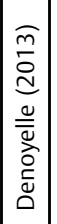 & 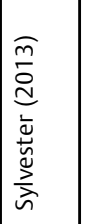 & 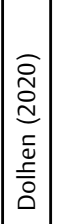 & 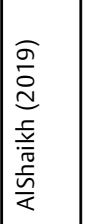 & 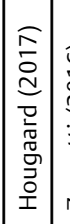 & 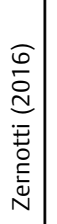 & & 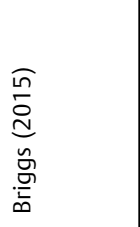 & 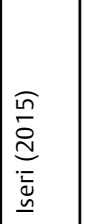 & 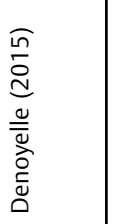 & 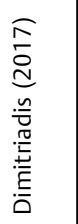 & 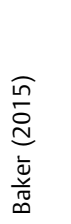 \\
\hline
\end{tabular}


12 studies focused specifically on children, and in 7 studies only adults were included.

\section{Results of the Integrative Review}

In the 37 articles included in the research, a total of 901 implantations were performed, of which 552 implanted Baha Attract devices, 244 implanted Bone Bridge, and 105 implanted Sophono. Regarding the type of implant reported, of the 37 articles under study, 14 reported exclusively on Baha Attract, 12 exclusively on BoneBridge, 8 exclusively on Sophono, and 3 articles addressed more than 1 type of prosthesis, including two reporting on both Baha Attract and Sophono, and one reporting on both BoneBridge and Sophono.

A total of 192 adverse events were reported, corresponding to a total complication rate of $21.3 \%$. Four (2\%) of these complications were due to either device malfunction or difficulty in adjusting the processor, and 188 (98\%) were associated with injuries to the patient directly or indirectly associated with the implant. Most complications in absolute terms occurred with the use of Baha Attract ( $n=135,70.3 \%$ ), but this was also the most used device in the series. However, taking into account the total complication rates, the device with the highest percentage of complications was Sophono (26.6\%), followed by the Baha Attract (24.4\%) and BoneBridge (11.8\%). This same distribution was observed when considering the rates of minor complications specific to each type of implant (Sophono 22.8\%, Baha Attract 22.1\%, BoneBridge $6.1 \%)$, but it was not observed when considering the rates of major complications specific to each type of implant (BoneBridge 5.7\%, Sophono 3.8\%, Baha Attract 2.3\%). - Table 1 describes the adverse events reported by device type. All reports of adverse events occurred in postoperative settings.

The most commonly reported adverse events were minor complications $(N=161)$, which corresponded to $84.3 \%$ of all complications and a rate of $17.8 \%$. These complications included mild pain, hyperemia, numbness, mild wound infection, and mild ulceration. None of these complications prevented the use of the hearing implant permanently, and all were resolved by reducing the strength of the magnet or decreasing the number of hours using the device per day, or even with local care.

Major complications $(N=31)$ were $16.1 \%$ of all complications. Their rate corresponded to $3.4 \%$ in the present series. Among these complications, the most frequent were wound infection or explantation ( $N=8,25.8 \%)$, skin necrosis, ulceration, or prolonged healing $(N=6,19.3 \%)$, and skin rupture $(N=5,16.1 \%)$. The remaining complications were grouped in -Table 2. All patients (100\%) who had major complications had to stop using the hearing implant.

\section{Discussion}

Transcutaneous bone-conduction devices emerged as an alternative to percutaneous devices, because they do not require an abutment crossing the skin to support the external processor and, therefore, have advantages from an aesthetic point of
Table 2 Summary of complications

\begin{tabular}{|l|l|}
\hline Major complications & $\mathrm{N}$ \\
\hline Seroma/hematoma & 1 \\
\hline Surgical wound infections/explantation & 8 \\
\hline Skin rupture & 5 \\
\hline Difficulty adjusting processor & 2 \\
\hline Device fault & 2 \\
\hline Necrosis/skin ulceration/prolonged healing & 6 \\
\hline Extrusion & 2 \\
\hline Compressive symptoms & 1 \\
\hline Refractory headache & 1 \\
\hline Tinnitus & 1 \\
\hline Disabling pain & 2 \\
\hline & 31 \\
\hline Minor complications & 162 \\
\hline $\begin{array}{l}\text { Mild pain, skin hyperemia, numbness, } \\
\text { mild surgical wound infection, mild ulceration }\end{array}$ & 193 \\
\hline Total & \\
\hline
\end{tabular}

view and regarding skin-related complications. Among the transcutaneous devices, active devices have an advantage over passive ones, as they require a lower-strength magnet to support themselves, and they also prevent the attenuation of vibrations produced by soft tissues. The aim of the present study is to document the complications associated with transcutaneous bone-conduction devices to assess the best treatment option for the patients.

The present review also showed that passive transcutaneous devices have higher complication rates than the active ones (Sophono 26.6\% and Baha Attract $24.4 \%$ versus BoneBridge $11.8 \%$ ). Some studies suggest that the complication rate for transcutaneous devices is around $35.7 \%$ for passive devices and $9.4 \%$ for active ones. ${ }^{19,20}$

The rate of minor complications was $17.8 \%$, with devicespecific rates of $22.8 \%$ for Sophono, $22.1 \%$ for Baha Attract, and $6.1 \%$ for BoneBridge. This is in agreement with the literature, which reports that BoneBridge offers a lower rate of complications, especially when compared with percutaneous prostheses. $^{21,22}$

With regard to the treatment of situations such as minor complications (hyperemia, skin edema, mild infection, pain), treatment was generally and predominantly clinical, progressing with local care and systemic oral antibiotic therapy. These complications were linked to, at most, momentary discontinuation of implant use, and more aggressive conducts, such as explantations, were not required. According to Kruyt (2020) and Gawecki (2019), in a prospective cohort in which Baha Attract was used, it was evidenced that most patients initially reported experiencing some degree of pain or discomfort and numbness. However, these complication rates decreased during follow-up, being reported only sporadically in the last follow-up consultation. ${ }^{23,24}$ 
In the present study, a lower complication rate of $22.8 \%$ was observed with the Sophono implant, and the most frequent complication described was a minor one, related to pain or pressure around the external unit, with the symptoms being relieved by decreasing magnet intensity, as previously reported by Shin (2016). ${ }^{25}$ However, some studies, such as the one by Marsella (2014), also report the persistence of this symptom, even with a decrease in the intensity of the magnet, making it unfeasible for the patient to use the device due to pain, which constitutes a major complication. ${ }^{26}$

Regarding the occurrence and treatment of major complications, in the present review, a complication rate of $5.7 \%$ was observed for BoneBridge, which is in agreement with literature studies, such as that by Brkic (2020), in which the rate of major complications with the use of BoneBridge was 9.4\% (6/64), including 5 explantations and 1 reimplantation. ${ }^{19}$ Similar results were also found in the study by Seiwerth (2021), ${ }^{27}$ in which the major complication rate was $6.2 \%$.

The overall rate of complications in the present series was $21.3 \%$, which compares favorably to the high rates of complications associated with percutaneous devices, which according to the literature can range from 8 to $59 \%$. Transcutaneous devices generally show a significant reduction in patient injuries when compared with percutaneous models. ${ }^{4,19,20,27-32}$

The present study has some limitations. Being a systematic review, it is limited by the individual methodology of the articles included, since there was no restriction regarding the study design of the articles included, which was reflected in the heterogeneity of the studies regarding the follow-up period, the audiological measures, and the reports of complications. There is no validated scale for complications resulting from transcutaneous devices. Therefore, it is difficult to define which minor complications, such as erythema or pain at the implant site, are clinically significant, and this category is presumed by the authors in cases in which the treatment was conservative, similarly to other published works. It is worth mentioning that the complication rates in the present work may be overestimated, due to the fact that the selected studies necessarily addressed complications,-whether major or minor,-and those studies that did not mention complications in their text were excluded.

\section{Final Comments}

Hearing implants offer a viable hearing alternative compared with percutaneous devices, and represent an alternative hearing resource for the patient. Although there is a frequency attenuation through soft tissue and its thickness, lower rates of postoperative complications and the aesthetics of a skin hearing implant make it an appealing option. Complications generally tend to be mild and managed clinically. However, major complications can occur, and it is essential that doctors are attentive to this possibility, especially in selected patients. Hence the importance of a well-reasoned, judicious indication, with a rigorous selection of patients.

\section{Conflict of Interests}

The authors have no conflict of interests to declare.

\section{References}

1 Berger KW. Early bone conduction hearing aid devices. Arch Otolaryngol 1976;102(05):315-318

2 Perciaccante A, Coralli A, Bauman NG. Beethoven: His hearing loss and his hearing aids. Otol Neurotol 2020;41(09):1305-1308

3 Kara A, Iseri M, Durgut M, Topdag M, Ozturk M. Comparing audiological test results obtained from a sound processor attached to a Softband with direct and magnetic passive bone conduction hearing implant systems. Eur Arch Otorhinolaryngol 2016;273(12):4193-4198

4 Ngui LX, Tang IP. Bonebridge transcutaneous bone conduction implant in children with congenital aural atresia: surgical and audiological outcomes. J Laryngol Otol 2018;132(08):693-697

5 Reinfeldt S, Håkansson B, Taghavi H, Eeg-Olofsson M. New developments in bone-conduction hearing implants: a review. Med Devices (Auckl) 2015;8:79-93

6 Dumper J, Hodgetts B, Liu R, Brandner N. Indications for boneanchored hearing AIDS: a functional outcomes study. J Otolaryngol Head Neck Surg 2009;38(01):96-105

7 Bento RF, Kiesewetter A, Ikari LS, Brito R. Bone-anchored hearing aid (BAHA): indications, functional results, and comparison with reconstructive surgery of the ear. Int Arch Otorhinolaryngol 2012; 16(03):400-405

8 Lavilla Martín de Valmaseda MJ, Cavalle Garrido L, Huarte Irujo A, et al. Clinical guideline on bone conduction implants. Acta Otorrinolaringol Esp 2019;70(02):105-111

9 Ellsperman SE, Nairn EM, Stucken EZ. Review of bone conduction hearing devices. audiology research. Audiology Res 2021;11(02): 207-219

10 Siau RT, Dhillon B, Siau D, Green KM. Bone-anchored hearing aids in conductive and mixed hearing losses: why do patients reject them? Eur Arch Otorhinolaryngol 2016;273(10):3117-3122

11 Dun CAJ, Faber HT, de Wolf MJF, Mylanus EA, Cremers CW, Hol MK. Assessment of more than 1,000 implanted percutaneous bone conduction devices: skin reactions and implant survival. Otol Neurotol 2012;33(02):192-198

12 Kiringoda R, Lustig LR. A meta-analysis of the complications associated with osseointegrated hearing aids. Otol Neurotol 2013;34(05):790-794

13 den Besten CA, Nelissen RC, Peer PG, et al. A retrospective cohort study on the influence of comorbidity on soft tissue reactions, revision surgery, and implant loss in bone-anchored hearing implants. Otol Neurotol 2015;36(05):812-818

14 Mohamad S, Khan I, Hey SY, Hussain SS. A systematic review on skin complications of bone-anchored hearing aids in relation to surgical techniques. Eur Arch Otorhinolaryngol 2016;273(03): 559-565

15 Marfatia H, Shah K, Shelke S, Krishnan K. Baha Attract: our experience. Indian J Otolaryngol Head Neck Surg 2017;69(04):544-548

16 Sharma S, Bulstrode N, Marshall A. Implantation and positioning of a transcutaneous magnetic bone conduction hearing system for children with microtia in preparation for auricular reconstruction. Clin Otolaryngol 2018;43(02):779-781

17 Hol MKS, Nelissen RC, Agterberg MJH, Cremers CW, Snik AF. Comparison between a new implantable transcutaneous bone conductor and percutaneous bone-conduction hearing implant. Otol Neurotol 2013;34(06):1071-1075

18 Iseri M, Orhan KS, Tuncer U, et al. Transcutaneous bone-anchored hearing aids versus percutaneous ones. Otol Neurotol 2015;36 (05):849-853 
19 Brkic FF, Riss D, Scheuba K, et al. Medical, technical and audiological outcomes of hearing rehabilitation with the bonebridge transcutaneous bone-conduction implant: A single-center experience. J Clin Med 2019;8(10):1614

20 O'Niel MB, Runge CL, Friedland DR, Kerschner JE. Patient outcomes in magnet based implantable auditory assist devices. JAMA Otolaryngol Head Neck Surg 2014;140(06):513-520

21 Zernotti ME, Chiaraviglio MM, Mauricio SB, Tabernero PA, Zernotti M, Di Gregorio MF. Audiological outcomes in patients with congenital aural atresia implanted with transcutaneous active bone conduction hearing implant. Int J Pediatr Otorhinolaryngol 2019;119:54-58

22 Zernotti ME, Di Gregorio MF, Galeazzi P, Tabernero P. Comparative outcomes of active and passive hearing devices by transcutaneous bone conduction. Acta Otolaryngol 2016;136(06):556-558

23 Kruyt IJ, Monksfield P, Skarzynski PH, et al. Results of a 2-year prospective multicenter study evaluating long-term audiological and clinical outcomes of a transcutaneous implant for bone conduction hearing. Otol Neurotol 2020;41(07):901-911

24 Gawęcki W, Balcerowiak A, Kalinowicz E, Wróbel M. Evaluation of surgery and surgical results of Baha ${ }^{\circledR}$ Attract system implantations - single centre experience of hundred twenty five cases. Rev Bras Otorrinolaringol (Engl Ed) 2019;85(05):597-602

25 Shin JW, Kim SH, Choi JY, et al. Surgical and audiologic comparison between sophono and bone-anchored hearing aids implantation. Clin Exp Otorhinolaryngol 2016;9(01):21-26
26 Marsella P, Scorpecci A, Vallarino MV, Di Fiore S, Pacifico C. Sophono in pediatric patients: The experience of an Italian tertiary care center. Otolaryngol Head Neck Surg 2014;151(02): 328-332

27 Seiwerth I, Fröhlich L, Schilde S, Götze G, Plontke SK, Rahne T. Clinical and functional results after implantation of the bonebridge, a semi-implantable, active transcutaneous bone conduction device, in children and adults. Eur Arch Otorhinolaryngol 2022 Jan;279(01):101-113

28 Hobson JC, Roper AJ, Andrew R, Rothera MP, Hill P, Green KM. Complications of bone-anchored hearing aid implantation. J Laryngol Otol 2010;124(02):132-136

29 Goycoolea M, Ribalta G, Tocornal F, et al. Clinical performance of the Osia ${ }^{\mathrm{TM}}$ system, a new active osseointegrated implant system. Results from a prospective clinical investigation. Acta Otolaryngol 2020;140(03):212-219

30 Sprinzl GM, Wolf-Magele A. The Bonebridge Bone Conduction Hearing Implant: indication criteria, surgery and a systematic review of the literature. Clin Otolaryngol 2016;41(02):131-143

31 Lin J, Chen S, Zhang H, et al. Application of Implantable Hearing Aids and Bone Conduction Implant System in patients with bilateral congenital deformation of the external and middle ear. Int J Pediatr Otorhinolaryngol 2019;119:89-95

32 Mylanus EAM, Hua $\mathrm{H}$, Wigren S, et al. Multicenter clinical investigation of a new active osseointegrated steady-state implant system. Otol Neurotol 2020;41(09):1249-1257 